\title{
Die Jodometrie des Thalliums als Chromat.
}

\author{
Von \\ E. RUPP.
}

Crookes ${ }^{1}$ hat dargethan, dafs durch Alkalidichromat aus alkalischen Thallosalzlösungen neutrales und beinahe unlösliches Thallochromat gefällt wird. Er machte hiervon Gebrauch zu einer ungefähren Trennung von Thallium und Cadmium. ${ }^{2}$

BRowning und HUTCHINs begründeten sodann eine brauchbare gravimetrische Bestimmungsmethode des Thalliums auf die Fällung als Chromat, ${ }^{3}$ indem sie die ammon- oder kaliumkarbonatalkalische Thallosalzlösung in der Wärme mit einem C̈berschusse von Kaliumdichromat fällen, den Niederschlag von Thallochromat über Asbest sammeln, waschen und bis zur Gewichtskonstanz trocknen.

Dieselben Autoren versuchten dann auch die Methode zu einer volumetrischen zu gestalten, indem sie von einer Dichromatlösung bekannten Gehaltes ausgeben, den nach der Fällung verbleibenden Überschufs hieran quantitativ vom Niederschlag trennen und mittels arseniger Säure ${ }^{4}$ bestimmen. Hierbei gelangen sie jedoch zu dem Ergebnis, dais ,wegen des sehr hohen Molekulargewichtes des Thallooxyds im Vergleich zum Molekulargewicht der Chromsäure diese letztere Methode natürlich nicht sehr genau sein kann" und die Resultate nur einigermafsen mit der gravimetrischen Methode übereinstimmen.

Der wunde Punkt bei der Bestimmung des Thalliums als Chro-

1 Chem. News 8, 255.

${ }^{2}$ Chem. News 7, 145.

3 Z. anorg. Chem. 22, 380.

- Am. Journ. Soc. 1, 35. 
mat liegt in der merkbaren Wasserlöslichkeit des letzteren (0.03 T'eile in 100 Teilen Wasser von $60^{\circ}$ ). Diese Schwierigkeit läfst sich mit der für Wismutchromatbestimmungen ${ }^{1}$ von mir eingeschlagenen Versuchsanordnung verhältnismälsig leicht dadurch vermeiden, dafs man das Chromatfällungsgemisch auf ein bekannntes Volum bringt und in einem aliquoten Filtratteile den $\mathrm{CrO}_{3}$-Überschuls jodometrisch zurückmifst. Indem hierbei der Niederschlag mit reiner Waschflüssigkeit überhaupt nicht in Berührung gelangt, die Konzentrationsverhältnisse beliebigt wählbar sind und die Löslichkeit des Chromatniederschlages im Fällungsgemische an und für sich schon eine herabgeminderte sein dürfte, so liefs sich annehmen, dafs auf diesem Wege die Bestimmung des Thalliums als Chromat volumetrisch mindestens ebenso gute Resultate liefern muls, wie gravimetrisch.

Die experimentelle Prüfung hierauf habe ich im Verein mit Herrn stud. ZimmLr ausgeführt. Als Fällungsmaterial bedienten wir uns durchweg des gelben Kaliumchromats.

Die betreffenden Versuche wurden mit einer Lösung reinsten krystallisierten Thalliumnitrats angestellt, die $0.25637 \mathrm{~g} \mathrm{TlNO}_{3}$ in $10 \mathrm{ccm}$ enthielt. Den Fällungsgemischen wurden verschiedenerlei Zusatzstoffe beigefügt, um den Reaktionsverlauf in neutraler, saurer und alkalischer Lösung kennen zu lernen, bezw. um festzustellen, unter welchen Bedingungen einheitliche Fällungen von Thallochromat oder Thallodichromat erbalten werden könnten.

Die Prüfung mulste sich aus den Titrationswerten nach folgenden Ansätzen ergeben:

$$
\begin{gathered}
2 \mathrm{TlNO}_{3}+\mathrm{K}_{2} \mathrm{CrO}_{4}=\mathrm{Tl}_{2} \mathrm{CrO}_{4}+2 \mathrm{KNO}_{3}, \\
2 \mathrm{TINO}_{3}=\mathrm{CrO}_{8}=3 \mathrm{~J} \\
2 \mathrm{TINO}_{3}=\frac{\mathrm{J}}{\overline{30}}=1000 \mathrm{~cm} n / 10 \text { Thiosulfat. } \\
0.01777 \mathrm{~g} \mathrm{TINO}{ }_{3}=0.01362 \mathrm{~g} \mathrm{Tl}=1 \mathrm{ccm} \text { Thiosulfat. } \\
2 \mathrm{TINO}_{3}+\mathrm{K}_{2} \mathrm{Cr}_{2} \mathrm{O}_{7}=\mathrm{Tl}_{2} \mathrm{Cr}_{2} \mathrm{O}_{7}+2 \mathrm{KNO}_{3}, \\
2 \mathrm{TINO}_{3}=2 \mathrm{CrO}_{3}=6 \mathrm{~J} \\
\mathrm{TINO}_{3}=\frac{\mathrm{J}}{10}=1000 \mathrm{ccm} n / 10 \text { Thiosulfat. }
\end{gathered}
$$

$0.008888 \mathrm{~g} \mathrm{TINO}_{3}=0.006814 \mathrm{~g} \mathrm{Tl}=1 \mathrm{ccm}$ Thiosulfat.

Z. anorg. Chem. 32, 362. 
Bei den in untenstehender Tabelle aufgeführten Analysen war so verfahren worden, dafs die Thalliumsalzlösung in $10 \mathrm{ccm}$ der mit den jeweiligen 'Zusätzen versehenen Kaliumchromatlösung bekannten Jodwertes unter Umschwenken eingegossen wurde. Das Volumen des Gemisches wurde sodann auf $100 \mathrm{ccm}$ ergänzt und das Ganze ca. $1 / 2$ Stunde stehen gelassen.

Zur nunmehrigen Filtration dienten zwei ineinander gelegte dichte Filter. Es lassen sich dadurch $25-50 \mathrm{ccm}$ klares Filtrat gewinnen, die zur Rückbestimmung überschüssigen Alkalichromats dienen.

Aus der hierfür erforderlichen bezw. auf das Gesamtvolumen von $100 \mathrm{ccm}$ umgerechneten Thiosulfatmenge und dem ursprünglichen Titer der Chromatlösung ergab sich durch Differenz der auf Thallium entfallende Thiosulfatwert.

\begin{tabular}{|c|c|c|c|c|c|c|}
\hline \multirow{2}{*}{ Nr. } & \multirow{2}{*}{$\begin{array}{l}\text { Ange- } \\
\text { wandte } \\
\text { Tl-Lösg. } \\
\text { in ccm }\end{array}$} & \multirow{2}{*}{ Zusatz } & \multicolumn{2}{|c|}{$n / 10$ Thiosulfatverbr. } & \multirow{2}{*}{$\begin{array}{c}\text { Grefund. } \\
\text { Tl-Menge } \\
\text { in } \%\end{array}$} & \multirow{2}{*}{$\begin{array}{l}\text { Berechneter } \\
\text { Thiosulfat- } \\
\text { verbrauch }\end{array}$} \\
\hline & & & $\begin{array}{l}\text { f. angew. } \\
\text { Tl-Menge }\end{array}$ & \begin{tabular}{|c|} 
pro $10 \mathrm{ecm}$ \\
Tl-Lösg.
\end{tabular} & & \\
\hline 1 & 20 & - & 28.55 & 14.28 & 98.9 & pro $10 \mathrm{~cm}$ \\
\hline 2 & 20 & - & 28.82 & 14.41 & 99.88 & Tl-Lösg. \\
\hline 3 & 20 & 5 g Na-Acetat & 28.60 & 14.3 & 99.12 & $14.43 \mathrm{cem}$ \\
\hline 4 & 10 & , & 14.42 & 14.42 & 99.05 & f. $\mathrm{TI}_{2} \mathrm{CrO}_{4}$. \\
\hline 5 & 10 & , & 14.57 & 14.57 & 100.09 & $28.86 \mathrm{cem}$ \\
\hline 6 & 10 & $\begin{array}{c}5 \mathrm{~g} \mathrm{Na} \text {-Acetat } \\
+5 \text { cem Essigss. }\end{array}$ & 22.2 & 22.2 & 76.96 & f. $\mathrm{Tl}_{2} \mathrm{Cr}_{2} \mathrm{O}_{7}$ \\
\hline 7 & 20 & , & 43.92 & 21.96 & 76.09 & - \\
\hline 8 & 20 & , & 40.48 & 20.24 & 70.13 & - \\
\hline 9 & 20 & $"$ & 44.8 & 22,4 & 77.61 & - \\
\hline 10 & 20 & $"$ & 41.68 & 20.84 & 72.21 & - \\
\hline 11 & 20 & $"$ & 38.8 & 19.4 & 67.22 & - \\
\hline 12 & 20 & , & 38.8 & 19.4 & 67.22 & - \\
\hline 13 & 20 & $5 \mathrm{ccm} \mathrm{NH} \mathrm{NH}_{3} 10 \%$ & 28.8 & 14.4 & 99.81 & - \\
\hline 14 & 20 &, & 28.8 & 14.4 & 99.81 & - \\
\hline 15 & 10 & $10 \mathrm{cem} \mathrm{NH} 30 \%$ & 14.42 & 14.42 & 99.95 & - \\
\hline 16 & 10 & $1 \mathrm{~g} \mathrm{CaCO}_{3}$ & 14.42 & 14.42 & 99.95 & - \\
\hline 17 & 10 &, & 14.38 & 14.38 & 99.67 & - \\
\hline 18 & 10 & $0.5 \mathrm{~g} \quad \mathrm{~K}_{2} \mathrm{CO}_{\mathrm{g}}$ & 14.39 & 14.39 & 99.74 & - \\
\hline 19 & 20 & $5 \mathrm{cem} \mathrm{H}_{2} \mathrm{SO}_{4}$ verd. & 57.44 & 28.72 & 99.53 & - \\
\hline 20 & 20 & $"$ & 57.44 & 28.72 & 99.53 & - \\
\hline
\end{tabular}

Die Versuche $1-5$ zeigen, dafs in neutraler Lösung sowohl ohne, als namentlich auch mit Zusatz von Natriumacetat befriedigende Resultate erzielt werden. 
Versuche 6-12 waren angestellt worden, um zu ermitteln, ob in solchen Fällen, wo mineralsaure Thalliumsalzlösungen vorliegen, durch Natriumacetat eine Neutralisation herbeigeführt werden kann, bezw: ob die Bestimmungen in essigsaurer Lösung ebenso verlaufen, wie in neutraler.

Wie sich zeigt, ist dies nicht der Fall. Vielmehr bestehen die Niederschläge aus wechselnden Mengen von Thallochromat und Dichromat, entsprechend den in der Kaliumchromatlösung durch die Essigsäure erzeugten Kaliumdichromatmengen.

Versuche 13-18 zeigen, wie bei sauren Thalliumsalzlösungen in einfacher Weise durch neutralisiẹrende Zusütze sehr genaue und konstante Resultate gewonnen werden können.

Am zweckdienlichsten hierfür war Calciumkarbonat, das sich infolge Einhüllungserscheinungen mit dem Thalliumchromat zu einem leicht und klar abfiltrierenden Niederschlag vereinigt, während die ammoniakalischen Gemische vielfach trübe filtrieren.

Aus Gründen der erleichterten Filtration wie der grölseren Konstanz ist deshalb stets ein Zusatz von Calciumkarbonat - auch in neutraler Lösung - empfehlenswert.

Die Vorsuche 19 und 20 der Tabelle zeigen, dafs aus genügend mineralsaurer Lösung Thalliumdichromat in einheitlicher Form gewonnen wird, und dals dieses hinreichend wasserunlöslich ist, um unter den angeführten Bedingungen gleichfalls analytisch verwertbar zul sein.

Die praktische Durchführung der Analyse gestaltet sich in kurzer Zusammenstellung wie folgt: 10 event. auch $20 \mathrm{ccm}$ einer $5 \%$ igen Kaliumchromatlösung genauestens ermittelten $\mathrm{CrO}_{3}$-Wertes werden in einem 50 oder $100 \mathrm{ccm}-K o l b e n$ mit etwa der gleichen Menge Wasser und $1-2 \mathrm{~g}$ gefälltem Calciumkarbonat versetzt. Sodann wird unter Umschwenken ein bekanntes, nicht unter $0.1 \mathrm{~g}$ Tl enthaltendes Volum der neutralen oder mit Soda annähernd neutralisierten Thalliumsalzlösung hinzugefügt. Nachdem mit Wasser auf die Marke aufgefüllt und wiederholt tüchtig durchgeschüttelt worden, lärst man ca. 30 Minuten absitzen, giefst durch ein Doppelfilter ab und bestimmt in $20-50 \mathrm{ccm}$ des Filtrates den $\mathrm{CrO}_{3}$-Überschufs zurück. Hierbei wie bei der Chromattiter-Bestimmung verfährt man derart, dafs in einem Stöpselglase mit ca. $100 \mathrm{ccm}$ Wasser verdünnt, und mit $10-15 \mathrm{ccm} 25 \%$ iger Salzsäure angesäuert wird. Nachdem man ca. $1 \mathrm{~g}$ Jodkalium hinzugefügt, läfst man 5 Minuten stehen und 
titriert dann das ausgeschiedene Jod unter Zusatz von Stärkelösung mittels $n / 10$-Thiosulfat.

Die Differenz zwischen ursprünglichem und schliefslich verbleibendem Thiosulfatwerte der angewandten Chromatmenge ist mit der Mafsnahme auf Thallium umzurechnen, dafs $1 \mathrm{ccm} n / 10-$ Thiosulfat $=0.01362 \mathrm{~g}$ Tl entspricht.

Über weitere Metalltitrationen mittels Chromsäure wird später berichtet werden.

Chem. Universitätslaboratorium (Phil. Abtlg.), Freiburg i. B.

Bei der Redaktion eingegangen am 10. November 1902. 